\title{
Consistent Weighted Average Flux of Well-Balanced TVD-RK Discontinuous Galerkin Method for Shallow Water Flows
}

\author{
Thida Pongsanguansin, ${ }^{1}$ Montri Maleewong, ${ }^{2}$ and Khamron Mekchay ${ }^{1}$ \\ ${ }^{1}$ Department of Mathematics and Computer Science, Chulalongkorn University, Bangkok 10330, Thailand \\ ${ }^{2}$ Department of Mathematics, Kasetsart University, Bangkok 10900, Thailand
}

Correspondence should be addressed to Montri Maleewong; montri.m@ku.ac.th

Received 26 March 2015; Revised 2 June 2015; Accepted 14 June 2015

Academic Editor: Javier Murillo

Copyright (c) 2015 Thida Pongsanguansin et al. This is an open access article distributed under the Creative Commons Attribution License, which permits unrestricted use, distribution, and reproduction in any medium, provided the original work is properly cited.

A well-balanced scheme with total variation diminishing Runge-Kutta discontinuous Galerkin (TVD-RK DG) method for solving shallow water equations is presented. Generally, the flux function at cell interface in the TVD-RK DG scheme is approximated by using the Harten-Lax-van Leer (HLL) method. Here, we apply the weighted average flux (WAF) which is higher order approximation instead of using the HLL in the TVD-RK DG method. The consistency property is shown. The modified wellbalanced technique for flux gradient and source terms under the WAF approximations is developed. The accuracy of numerical solutions is demonstrated by simulating dam-break flows with the flat bottom. The steady solutions with shock can be captured correctly without spurious oscillations near the shock front. This presents the other flux approximations in the TVD-RK DG method for shallow water simulations.

\section{Introduction}

Hyperbolic balanced law for one-dimensional problem is

$$
U_{t}+F_{x}(U)=G(U)
$$

where $U, F$, and $G$ represent solution vector, flux function, and source terms, respectively.

In this work, the hyperbolic equation is the shallow water equations which can be expressed by

$$
\begin{aligned}
h_{t}+q_{x} & =0, \\
q_{t}+\left(\frac{q^{2}}{h}+\frac{g h^{2}}{2}\right)_{x} & =-g h b_{x},
\end{aligned}
$$

where $h$ is the water depth, $q=u h$ is the discharge, $u$ is the flow velocity in the $x$-direction, $g$ is the acceleration due to gravity, and $b$ is the bottom function. Equation (2) can be rewritten by setting

$$
\begin{aligned}
U & =\left(\begin{array}{l}
h \\
q
\end{array}\right), \\
F(U) & =\left(\begin{array}{c}
q \\
\frac{q^{2}}{h}+\frac{g h^{2}}{2}
\end{array}\right), \\
G(U) & =\left(\begin{array}{c}
0 \\
-g h b_{x}
\end{array}\right) .
\end{aligned}
$$

The total variation diminishing Runge-Kutta discontinuous Galerkin (TVD-RK DG) method can be applied to solve the shallow water equations; see [1-5]. This scheme has several advantages. For instance, it can be used to handle complex geometries, and in the same time, adaptive strategies are easily applied. The accuracy of numerical solutions can be improved by increasing the polynomial degree of 
approximating polynomial and by the method for estimating the flux function at cell interface.

By the concept of discontinuous Galerkin method, numerical solutions need not to be continuous at cell interface. So, we require an efficient flux approximation. Generally, there are several types of approximations. The most famous one is the Harten-Lax-van Leer flux (HLL), while the higher order approximation is the weighted average flux (WAF); see [6-9]. This approach approximates the flux functions by averaging flux in each direction along the wave solutions at the half-time step. It originates from random flux scheme, which was shown to be second-order accuracy in space and time in statistical sense by Toro [6]. From previous work, the weighted average flux has been successfully applied to solve various types of problems, especially in the finite volume method $[7,10,11]$. It improves the accuracy of the finite volume method to be second-order without reconstruction process. But the weighted average flux is rarely applied in the discontinuous Galerkin method. So the main objective of this work is the application of the WAF method in the TVD-RK DG method. We have also shown that the present scheme has consistent property with the WAF approximation.

The steady solutions of (3) can be obtained by setting

$$
\begin{aligned}
h u & =\text { constant } \\
\frac{1}{2} u^{2}+g(h+b) & =\text { constant. }
\end{aligned}
$$

Flux gradients are nonzero, and it must be balanced with the bottom gradient. Usually, numerical schemes without balancing these two quantities produce oscillate steady solution. So a balancing numerical scheme is needed, and it is called the well-balanced scheme. This method is designed to preserve energy at steady state. Generally, a numerical scheme is said to be well-balanced if it satisfies the exact C-property which was first introduced by Bermudez and Vazquez [12]. The exact Cproperty means that the numerical solution must satisfy still water condition at steady state given by

$$
\begin{aligned}
u & =0, \\
h+b & =\text { constant } .
\end{aligned}
$$

Thus, to obtain a well-balanced scheme, we must design the method that its steady solutions satisfy (5).

Recently, the second-order Runge-Kutta discontinuous Gelerkin method with well-balanced scheme is proposed by Kesserwani and Liang [13]. They presented the wetting and drying algorithms for solving one-dimensional problem. Xing and Shu [14] proposed a well-balanced finite volume method based on the weighted essentially nonoscillatory (WENO) scheme for solving one- and two-dimensional problems. They applied the decomposing source term technique into the sum of several terms to ensure the numerical balance between flux difference and source terms. Audusse et al. [15] presented a well-balanced finite volume with hydrostatic reconstruction to solve one-dimensional problem. Later, Noelle et al. [16] extended the well-balanced finite volume schemes proposed by [15] to any that desired the orders of accuracy.
In order to solve the shallow water equations with source terms, we modified the well-balanced discontinuous Galerkin scheme proposed by [15] and related work by Xing and Shu [14]. The weighted average flux (WAF) method [6, 7] has been applied in the modified DG scheme. We will show that the modified TVD-RK DG scheme with the WAF has consistent property. Various numerical experiments for both steady and unsteady flows are demonstrated to show the capability and accuracy of our numerical scheme.

Numerical scheme and its consistency property with WAF for the TVD-RK DG method have been shown in Section 2. The modified well-balanced TVD-RK DG scheme with source terms is presented in Section 3. Numerical examples are demonstrated in Section 4. Conclusions are finally made in Section 5.

\section{Numerical Scheme for Shallow Water Equations without Source Term}

The TVD-RK DG with weighted average flux method for the case of without source terms is presented in this section.

2.1. TVD-RK Discontinuous Galerkin Method. Consider the one-dimensional hyperbolic conservation law,

$$
U_{t}+F_{x}(U)=0
$$

The computational domain $(0, L)$ is divided into $K$ cells. We denote the $j$ th cell by $I_{j}=\left[x_{j-1 / 2}, x_{j+1 / 2}\right]$, for $j=1, \ldots, K$, with uniform cell size $\Delta_{j}=x_{j+1 / 2}-x_{j-1 / 2}$. The cell center is that $x_{j}=\left(x_{j+1 / 2}+x_{j-1 / 2}\right) / 2$, where $x_{j+1 / 2}$ and $x_{j-1 / 2}$ are the left and the right of cell boundaries, respectively.

Approximate solution is denoted by $U_{h}=\left(\begin{array}{l}h_{h} \\ q_{h}\end{array}\right)$.

Multiplying (6) by a test function, $v_{h}(x) \in P^{N}\left(I_{j}\right)$, where $P^{N}\left(I_{j}\right)$ is the polynomial space degree $N$ on the interval $I_{j}$, and replacing $U$ by $U_{h}$ and then taking the integration by parts over $I_{j}$, we obtain a weak form of numerical scheme as

$$
\begin{aligned}
& \int_{I_{j}}\left(\partial_{t} U_{h}\right) v_{h} d x-\int_{I_{j}} F\left(U_{h}\right) \partial_{x} v_{h} d x+\widehat{F}_{j+1 / 2} v_{h}\left(x_{j+1 / 2}^{-}\right) \\
& \quad-\widehat{F}_{j-1 / 2} v_{h}\left(x_{j-1 / 2}^{+}\right)=0
\end{aligned}
$$

where the flux function $F$ at the cell interfaces is approximated by $\widehat{F}$, which is the function of $U_{h}^{+}$and $U_{h}^{-}$at $x_{j \pm 1 / 2}$ as

$$
\widehat{F}_{j \pm 1 / 2}=\widehat{F}\left(\left(U_{h}\right)_{j \pm 1 / 2}^{-},\left(U_{h}\right)_{j \pm 1 / 2}^{+}\right) .
$$

Here $\left.U_{h}\right|_{j \pm 1 / 2} ^{-}$and $\left.U_{h}\right|_{j \pm 1 / 2} ^{+}$denote the approximate solutions at the left and the right of cell boundaries, respectively. If we apply the Legendre polynomials to be a local basis function, the approximate solution $U_{h}$ can be written by

$$
U_{h}(x, t)=\sum_{m=0}^{N} U_{j}^{m}(t) \varphi_{m}(x),
$$

where $U_{j}^{m}(t)$ is called the temporal coefficient. Now, the basis function $\varphi_{m}(x)$ is defined by the Legendre polynomial $P_{m}(x)$ of degree $m$ over $[-1,1]$. 
The test function $v_{h}(x)$ is typically chosen to be the basis function; that is, $v_{h}(x)=\left\{\varphi_{l}(x)\right\}_{l=0}^{N}$. So after applying Legendre's properties, (7) is simplified to

$$
\begin{aligned}
\frac{d U_{j}^{l}(t)}{d t}= & \frac{2 l+1}{\Delta_{j}} \int_{I_{j}} F\left(U_{h}\right) \partial_{x} \varphi_{l}(x) d x \\
& +\frac{2 l+1}{\Delta_{j}}\left\{(-1)^{l} \widehat{F}_{j-1 / 2}-\widehat{F}_{j+1 / 2}\right\},
\end{aligned}
$$

for $j=1, \ldots, K$, and $l=0, \ldots, N$.

The time derivative term in (10) can be approximated by applying the high-order TVD Runge Kutta (TVD-RK) method; see $[1,3,17]$. Note that, when the polynomial degree $N$ is applied, the TVD-RK method at least the order of $N+1$ must be applied to obtain the accuracy of order $O\left(\Delta x^{N+1}\right)$ for smooth flows.

The TVD-RK DG method can be used to simulate shallow water flows with moving shocks. Unphysically oscillate behaviours are usually produced near the shock fronts. The slope limiter techniques can be applied to remove the oscillations. In this work, we apply the Monotonic UpstreamCentered Scheme for Conservation Laws (MUSCL) limiter (see $[1,3,6,18]$ ) in the TVD-RK DG method. This approach limits the present solution slope by comparing the slope with neighbor cells.

2.2. Weighted Average Flux (WAF). The HLL numerical flux (Harten-Lax-van Leer) is usually used in the TVD-RK DG method. Another choice but higher order is the weighted average flux (WAF). It was first introduced by Toro; see $[6,7]$. The WAF approximation is second-order accurate in both space and time in statistical sense [6]. This approximation has been extensively applied in the finite volume method, but it is rarely used in the TVD-RK DG method. So the main objective of this work is to try to modify the WAF in the TVDRK DG method.

The weighted average flux, $\widehat{F}^{\mathrm{WAF}}$ at the interface, $x_{j+1 / 2}$, is defined by the integral average of a flux function $F(U)$ at the half-time step,

$$
\widehat{F}_{j+1 / 2}^{\mathrm{WAF}}=\frac{1}{\Delta x} \int_{-\Delta x / 2}^{\Delta x / 2} F\left(U_{j+1 / 2}\left(x, \frac{\Delta t}{2}\right)\right) d x .
$$

It can be written in the wave structure form as

$$
\widehat{F}_{j+1 / 2}^{\mathrm{WAF}}=\sum_{k=1}^{N_{c}+1} \omega_{k} F_{j+1 / 2}^{(k)}
$$

where $N_{c}$ is the number of wave solutions in the Riemann problem and $F_{j+1 / 2}^{(k)}$ is the $k$ th flux of the Riemann problem. For one-dimensional shallow water flows, we have $N_{c}=2$, where $F_{j+1 / 2}^{(1)}=F\left(U_{j+1 / 2}^{-}\right)$and $F_{j+1 / 2}^{(3)}=F\left(U_{j+1 / 2}^{+}\right)$. Flux component $F_{j+1 / 2}^{(2)}$ can be obtained from the HLL approach [6]. Weighted values, $\omega_{k}$, are defined by $\omega_{k}=(1 / 2)\left(c_{k}-c_{k-1}\right)$, where $c_{k}=S_{k} \Delta t / \Delta x$ is the Courant number of wave $k, c_{0}=$ $-1, c_{N_{c}+1}=1$, and $S_{k}$ is the speed of wave $k$.
To avoid spurious oscillations near a shock front, the WAF method will be modified by enforcing a total variation diminishing (TVD) scheme $[6,7,9,11,19]$. The TVD-WAF version is that

$$
\begin{aligned}
\widehat{F}_{j+1 / 2}^{\mathrm{WAF}-\mathrm{TVD}}= & \frac{1}{2}\left(F\left(U_{j+1 / 2}^{-}\right)+F\left(U_{j+1 / 2}^{+}\right)\right) \\
& -\frac{1}{2} \sum_{k=1}^{N_{c}} \operatorname{sgn}\left(c_{k}\right) \phi_{j+1 / 2}^{(k)} \Delta F_{j+1 / 2}^{(k)},
\end{aligned}
$$

where

$$
\Delta F_{j+1 / 2}^{(k)}=F_{j+1 / 2}^{(k+1)}-F_{j+1 / 2}^{(k)} .
$$

Here $\phi_{j+1 / 2}^{(k)}$ is a WAF limiter function. There are various choices; see more details in $[6,7,9,11,19]$. In this work, we choose the basic one of minmod type.

2.3. Consistency of WAF with TVD-RK DG Method. In this section, we show the consistency of the weighted average flux in the TVD-RK discontinuous Galerkin method.

Lemma 1. The TVD Runge-Kutta discontinuous Galerkin method is consistent with the weighted average flux for smooth flows.

Proof. Considering the weak form of RKDG method for the conservation law,

$$
\begin{aligned}
\int_{I_{j}} & \left(\frac{\partial}{\partial t} U\right) v(x) d x-\int_{I_{j}} F(U) v^{\prime}(x) d x \\
& -F\left(U_{j+1 / 2}\right) v\left(x_{j+1 / 2}^{-}\right)+F\left(U_{j-1 / 2}\right) v\left(x_{j-1 / 2}^{+}\right) \\
\quad= & 0 .
\end{aligned}
$$

Solution $U$ is approximated by $U_{h}^{*}$. The test function $v$ is estimated by $v_{h}$. Flux function at cell interfaces is approximated in terms of numerical flux, $\widehat{F}_{j \pm 1 / 2}$, so we obtain a numerical scheme of the following form:

$$
\begin{aligned}
\int_{I_{j}}( & \left.\frac{\partial}{\partial t} U_{h}^{*}\right) v_{h}(x) d x-\int_{I_{j}} F\left(U_{h}^{*}\right) v_{h}^{\prime}(x) d x \\
& -\widehat{F}_{j+1 / 2}\left(\left(U_{h}^{*}\right)_{j+1 / 2}^{-},\left(U_{h}^{*}\right)_{j+1 / 2}^{+}\right) v_{h}\left(x_{j+1 / 2}^{-}\right) \\
& +\widehat{F}_{j-1 / 2}\left(\left(U_{h}^{*}\right)_{j-1 / 2}^{-},\left(U_{h}^{*}\right)_{j-1 / 2}^{+}\right) v_{h}\left(x_{j-1 / 2}^{+}\right)=0 .
\end{aligned}
$$

Substituting $U_{h}^{*}$ by $U$ in (16) and subtracting with (15), we obtain a truncation error term $T_{1}$ as

$$
\begin{aligned}
T_{1} & =\mid \int_{I_{j}} \frac{\partial}{\partial t}[U-U] v_{h}(x) d x \\
& -\int_{I_{j}}[F(U)-F(U)] v_{h}^{\prime}(x) d x
\end{aligned}
$$




$$
\begin{aligned}
& -\left[F\left(U_{j+1 / 2}\right)-\widehat{F}_{j+1 / 2}\left(U_{j+1 / 2}^{-}, U_{j+1 / 2}^{+}\right)\right] v_{h}\left(x_{j+1 / 2}^{-}\right) \\
& +\left[F\left(U_{j-1 / 2}\right)-\widehat{F}_{j-1 / 2}\left(U_{j-1 / 2}^{-}, U_{j-1 / 2}^{+}\right)\right] \\
& \cdot v_{h}\left(x_{j-1 / 2}^{+}\right)
\end{aligned}
$$

When the solution is smooth, it must be continuous at $x_{j+1 / 2}$ or $U_{j+1 / 2}^{+}=U_{j+1 / 2}^{-}=U_{j+1 / 2}$.

The TVD weighted average flux is defined by

$$
\begin{aligned}
\widehat{F}_{j+1 / 2} & \left(U_{j+1 / 2}^{-}, U_{j+1 / 2}^{+}\right) \\
= & \frac{1}{2}\left(F\left(U_{j+1 / 2}^{-}\right)+F\left(U_{j+1 / 2}^{+}\right)\right) \\
& -\frac{1}{2} \sum_{k=1}^{2} \operatorname{sgn}\left(c_{k}\right) \phi^{k} \Delta F^{(k)},
\end{aligned}
$$

where $F^{(1)}=F\left(U_{j+1 / 2}^{-}\right)=F\left(U_{j+1 / 2}\right), F^{(3)}=F\left(U_{j+1 / 2}^{+}\right)=$ $F\left(U_{j+1 / 2}\right)$, and the component $F^{(2)}=F\left(U_{j+1 / 2}\right)$ which can be obtained from the HLL method $[4,6,7]$. Thus,

$$
\begin{aligned}
\widehat{F}_{j+1 / 2} & \left(U_{j+1 / 2}^{-}, U_{j+1 / 2}^{+}\right) \\
= & \frac{1}{2}\left(F\left(U_{j+1 / 2}\right)+F\left(U_{j+1 / 2}\right)\right) \\
& -\frac{1}{2} \sum_{k=1}^{2} \operatorname{sgn}\left(c_{k}\right) \phi^{k} \Delta F^{(k)}=F\left(U_{j+1 / 2}\right) .
\end{aligned}
$$

Similarly, we have $\widehat{F}_{j-1 / 2}\left(U_{j-1 / 2}^{-}, U_{j-1 / 2}^{+}\right)=F\left(U_{j-1 / 2}\right)$. Then, the weighted average flux is consistent.

For the TVD-RK DG method, the approximate solution $U_{h}^{*}$ in (16) is defined by

$$
U_{h}^{*}(x, t)=\sum_{k=0}^{N} U_{k}(t) \varphi_{k}(x)
$$

where $\varphi_{k}(x)$ is the Legendre polynomial degree $k$. Since the considering solution is smooth, we know from Theorem (3.1) in [1] that

$$
\left\|U-U_{h}^{*}\right\|_{L^{2}(0,1)} \leq O\left(\Delta x^{N+1 / 2}\right), \quad \text { for } N \geq 0 .
$$

This norm is defined as a truncation error term due to approximating polynomial, denoted by $T_{2}$.

After substituting the approximate solution into the numerical scheme (16), we obtain an ODE system,

$$
\frac{d}{d t} U_{h}^{*}=L\left(U_{h}^{*}\right)
$$

Next, we apply the TVD Runge-Kutta scheme for integrating in time. We approximate $U_{h}^{*}$ at each time step by $\widehat{U}_{h}$. The order of accuracy for time integration depends on the TVDRK order $[1,3,17]$. If the TVD-RK order $N+1$ is applied, a truncation error term $T_{3}$ is given by

$$
T_{3}=\left|U_{h}^{*}-\widehat{U}_{h}\right| \leq O\left(\Delta t^{N+1}\right) .
$$

Combining all of the truncation error terms together, the total truncation error term $T$ is estimated by

$$
T=T_{1}+T_{2}+T_{3} \leq O\left(\Delta x^{N+1 / 2}, \Delta t^{N+1}\right) .
$$

The total truncation error term is approaching zero as $\Delta x \rightarrow$ 0 and $\Delta t \rightarrow 0$. Hence, the TVD-RK DG with the WAF method is consistent.

\section{Well-Balanced TVD-RK DG with WAF Scheme}

In this section, we develop a well-balanced scheme for the TVD-RK DG with the WAF method. The main purpose is to present a modified scheme for solving the shallow water equations with source term. Also, this scheme must preserve exactly stationary solution when bottom slope exists. Let us start by considering the standard TVD-RK DG method,

$$
\begin{aligned}
& \int_{I_{j}}\left(\partial_{t} U_{h}\right) v_{h} d x-\int_{I_{j}} F\left(U_{h}\right) \partial_{x} v_{h} d x \\
& \quad+\widehat{F}_{j+1 / 2} v_{h}\left(x_{j+1 / 2}^{-}\right)-\widehat{F}_{j-1 / 2} v_{h}\left(x_{j-1 / 2}^{+}\right) \\
& =\int_{I_{j}} G\left(U_{h}\right) v_{h} d x,
\end{aligned}
$$

with initial condition

$$
\int_{I_{j}} U_{h}(x, 0) v_{h}(x) d x=\int_{I_{j}} U_{0}(x) v_{h}(x) d x
$$

where $\widehat{F}_{j \pm 1 / 2}$ are the weighted average flux in (13). Source term is given by

$$
G(U)=\left(\begin{array}{c}
G_{1}(U, x) \\
G_{2}(U, x)
\end{array}\right)=\left(\begin{array}{c}
0 \\
-g h b_{x}
\end{array}\right)
$$

We will derive a well-balanced scheme based on [14], but the weighted average flux is applied in the TVD-RK DG instead of using the Lax-Friedrichs (LF) flux. The main modification is the source term treatments in the WAF method. Assume that the source terms can be written by the summation of some functions as

$$
G_{p}(U, x)=\sum_{i} s_{i}(a(U, x)) t_{i}^{\prime}(x), \quad p=1,2,
$$


where $s_{i}$ and $t_{i}$ are some functions which will be determined later.

If the solution at steady state is stationary, then $a(U, x)$ in (28) can be decomposed by $a_{1}(U, x)$ and $a_{2}(U, x)$, such that

$$
\begin{aligned}
& a_{1} \equiv h+b=\text { constant, } \\
& a_{2} \equiv u=0 .
\end{aligned}
$$

Since $G_{1}$ is zero, we consider only $G_{2}$,

$$
G_{2}(U, x)=-g h b_{x}=-g(h+b) b_{x}+\frac{1}{2} g\left(b^{2}\right)_{x} .
$$

From (28), we have that

$$
\begin{aligned}
& s_{1}=s_{1}\left(a_{1}\right)=-g(h+b), \\
& s_{2}=\frac{1}{2} g,
\end{aligned}
$$

$$
\begin{aligned}
& \int_{I_{j}} G_{2}\left(U_{h}, x\right) v_{h} d x=\int_{I_{j}} \sum_{i} s_{i}(a(U, x)) t_{i}^{\prime}(x) v_{h}(x) d x \\
& =\sum_{i}\left[\frac{1}{2}\left(s_{i}\left(a\left(U_{h}, x\right)_{j-1 / 2}^{+}\right)+s_{i}\left(a\left(U_{h}, x\right)_{j+1 / 2}^{-}\right)\right)\right]\left[t_{i}\left(x_{i+1 / 2}\right) v_{h}\left(x_{i+1 / 2}^{-}\right)-t_{i}\left(x_{i-1 / 2}\right) v_{h}\left(x_{i-1 / 2}^{+}\right)-\int_{I_{j}} t_{i}(x) v_{h}^{\prime}(x) d x\right] \\
& \quad+\sum_{i}\left[\int_{I_{j}}\left\{s_{i}\left(a\left(U_{h}, x\right)\right)-\frac{1}{2}\left(s_{i}\left(a\left(U_{h}, x\right)_{j-1 / 2}^{+}\right)+s_{i}\left(a\left(U_{h}, x\right)_{j+1 / 2}^{-}\right)\right)\right\} t_{i}^{\prime}(x) v_{h}(x) d x\right] .
\end{aligned}
$$

Functions $a\left(u_{h}, x\right)$ and $t_{i}$ on the RHS of (34) can be approximated by $a_{h}\left(U_{h}, x\right)$ and $\left(t_{i}\right)_{h}$, where $a_{h}\left(U_{h}, x\right)$ and $\left(t_{i}\right)_{h}$ are the $L^{2}$ projection of $a\left(U_{h}, x\right)$ and $t_{i}$ into the space of $V_{h}$. Then, we obtain $\left(t_{1}\right)_{h}=b_{h}$ and $\left(t_{2}\right)_{h}=b_{h}^{2}$. Thus,

$$
F\left(U_{h}(x)\right)-\sum_{i} s_{i}\left(a\left(U_{h}, x\right)\right)\left(t_{i}(x)_{h}\right)=\alpha .
$$

Then,

$$
\begin{aligned}
\int_{I_{j}} G_{2}\left(U_{h}, x\right) v_{h} d x= & \sum_{i}\left[\frac{1}{2}\left(s_{i}\left(a_{h}\left(U_{h}, x\right)_{j-1 / 2}^{+}\right)+s_{i}\left(a_{h}\left(U_{h}, x\right)_{j+1 / 2}^{-}\right)\right)\right] \\
& \cdot\left[\left(\widehat{t}_{i}\right)_{h, j+1 / 2} v_{h}\left(x_{i+1 / 2}^{-}\right)-\left(\widehat{t}_{i}\right)_{h, j-1 / 2}\left(x_{i-1 / 2}\right) v_{h}\left(x_{i-1 / 2}^{+}\right)-\int_{I_{j}}\left(t_{i}\right)_{h}(x) v_{h}^{\prime}(x) d x\right] \\
& +\sum_{i}\left[\int_{I_{j}}\left\{s_{i}\left(a_{h}\left(U_{h}, x\right)\right)-\frac{1}{2}\left(s_{i}\left(a_{h}\left(U_{h}, x\right)_{j-1 / 2}^{+}\right)+s_{i}\left(a_{h}\left(U_{h}, x\right)_{j+1 / 2}^{-}\right)\right)\right\}\left(t_{i}\right)_{h}^{\prime}(x) v_{h} d x\right] .
\end{aligned}
$$

To satisfy the weighted average flux in (12), $\left(\widehat{t}_{i}\right)_{h, j+1 / 2}$ must be modified. Here, we assume that

$$
\left(\widehat{t}_{i}\right)_{h, j+1 / 2}=\sum_{k=1}^{N_{c}+1} \omega_{k} t_{h, j+1 / 2}^{(k)}
$$

or if the TVD version in (13) is applied, this term can be written by 
Note that $\omega_{k}$ are weighted values in the WAF approximations and $\phi^{(k)}$ is the WAF limiter function. So $t_{h, j+1 / 2}^{(k)}$ can be defined similar to $F_{j+1 / 2}^{(k)}$.
For one-dimensional shallow water equations, we have $N_{c}=2$, where $t_{h, j+1 / 2}^{(1)}=t_{h}\left(x_{j+1 / 2}^{-}\right)$and $t_{h, j+1 / 2}^{(3)}=t_{h}\left(x_{j+1 / 2}^{+}\right)$. The approximation of $t_{h, j+1 / 2}^{(2)}$ in the intermediate region can be obtained by the HLL approach,

$$
t_{h, j+1 / 2}^{(2)}= \begin{cases}t_{h}\left(x_{j+1 / 2}^{-}\right), & \text {if } S_{L} \geq 0, \\ \frac{S_{R} t_{h}\left(x_{j+1 / 2}^{-}\right)-S_{L} t_{h}\left(x_{j+1 / 2}^{+}\right)+S_{R} S_{L}\left(t_{h}\left(x_{j+1 / 2}^{+}\right)-t_{h}\left(x_{j+1 / 2}^{-}\right)\right)}{S_{R}-S_{L}}, & \text { if } S_{L}<0<S_{R}, \\ t_{h}\left(x_{j+1 / 2}^{+}\right), & \text {if } S_{R} \leq 0 .\end{cases}
$$

Here $S_{L}$ and $S_{R}$ are signal velocities in the Riemann problem.

At steady state, the solution is assumed to be stationary. It implies that $h+b=c$, where $c$ is constant and $u=0$. From (35), we have that

$$
\begin{aligned}
F & \left(U_{h}\right)-\sum_{i} s_{i}\left(a_{h}(U, x)\right) t_{i}(x) \\
& =\frac{1}{2} g\left(h_{h}\right)^{2}-\left[\frac{1}{2} g b_{h}^{2}-g\left(h_{h}+b_{h}\right) b_{h}\right] \\
& =\frac{1}{2} g\left(h_{h}+b_{h}\right)^{2}=\frac{1}{2} g c^{2}=\alpha .
\end{aligned}
$$

This shows the suitable choices of $a\left(U_{h}, x\right)$ and $t_{i}$ in the TVDRK DG with the WAF approximation.

Next, we will show that the TVD-RK DG with the WAF method preserves the well-balanced property.

Proposition 2. The TVD-RK DG with the WAF scheme has preserved exactly stationary solutions at steady state.

Proof. Since $a_{h}\left(U_{h}, x\right)$ and $a_{h}\left(U_{h}, x\right)_{i \mp 1 / 2}^{ \pm}$are equal to the same constant $c$ at each Gauss-Lobatto point over cell $I_{j}$, thus

$$
\sum_{i}\left[\int_{I_{j}}\left\{s_{i}\left(a_{h}\left(U_{h}, x\right)\right)-\frac{1}{2}\left(s_{i}\left(a_{h}\left(U_{h}, x\right)_{j-1 / 2}^{+}\right)+s_{i}\left(a_{h}\left(U_{h}, x\right)_{j+1 / 2}^{-}\right)\right)\right\}\left(t_{i}\right)_{h}^{\prime}(x) v_{h} d x\right]=0
$$

Hence, we obtain a truncation error term $T$ as

$$
\begin{aligned}
T & =\int_{I_{j}} F\left(U_{h}(x, t)\right) v_{h}^{\prime}(x) d x-\widehat{F}_{j+1 / 2} v_{h}\left(x_{j+1 / 2}^{-}\right) \\
& +\widehat{F}_{j-1 / 2} v_{h}\left(x_{j-1 / 2}^{+}\right)+\sum_{i}\left[\frac { 1 } { 2 } \left(s_{i}\left(a_{h}\left(U_{h}, x\right)_{j-1 / 2}^{+}\right)\right.\right. \\
& \left.\left.+s_{i}\left(a_{h}\left(U_{h}, x\right)_{j+1 / 2}^{-}\right)\right)\right]\left[\left(\widehat{t}_{i}\right)_{h, j+1 / 2} v_{h}\left(x_{i+1 / 2}^{-}\right)\right. \\
& -\left(\widehat{t}_{i}\right)_{h, j-1 / 2}\left(x_{i-1 / 2}\right) v_{h}\left(x_{i-1 / 2}^{+}\right) \\
& \left.-\int_{I_{j}}\left(t_{i}\right)_{h}(x) v_{h}^{\prime}(x) d x\right] .
\end{aligned}
$$

Using $\widehat{F}$ in (12), $\left(\hat{t}_{i}\right)$ in (37), and $a_{h}\left(U_{h}, x\right)_{j \mp 1 / 2}^{ \pm}=c$, we have that

$$
\begin{aligned}
T & =\int_{I_{j}}\left[F\left(U_{h}(x, t)\right)-\sum_{i} s_{i}(c)\left(t_{i}\right)_{h}\right] v_{h}^{\prime}(x) d x \\
& -\left[\sum_{k=1}^{3} \omega_{k} F_{j+1 / 2}^{(k)}-\sum_{i} s_{i}(c)\left(\sum_{k=1}^{3} \omega_{k} t_{h, j+1 / 2}^{(k)}\right)\right] \\
& \cdot v_{h}\left(x_{j+1 / 2}^{-}\right)
\end{aligned}
$$

$$
\begin{aligned}
& +\left[\sum_{k=1}^{3} \omega_{k} F_{j-1 / 2}^{(k)}-\sum_{i} s_{i}(c)\left(\sum_{k=1}^{3} \omega_{k} t_{h, j-1 / 2}^{(k)}\right)\right] \\
& \cdot v_{h}\left(x_{j-1 / 2}^{+}\right) \\
& =\int_{I_{j}}\left[F\left(U_{h}(x, t)\right)-\sum_{i} s_{i}(c)\left(t_{i}\right)_{h}\right] v_{h}^{\prime}(x) d x \\
& -\left[\sum_{k=1}^{3} \omega_{k}\left(F_{j+1 / 2}^{(k)}-\sum_{i} s_{i}(c) t_{h, j+1 / 2}^{(k)}\right)\right] v_{h}\left(x_{j+1 / 2}^{-}\right) \\
& +\left[\sum_{k=1}^{3} \omega_{k}\left(F_{j-1 / 2}^{(k)}-\sum_{i} s_{i}(c) t_{h, j-1 / 2}^{(k)}\right)\right] v_{h}\left(x_{j-1 / 2}^{+}\right) .
\end{aligned}
$$

After applying condition (35) and rearranging terms, yields

$$
\begin{aligned}
T= & \alpha\left[v_{h}\left(x_{j+1 / 2}^{-}\right)-v_{h}\left(x_{j-1 / 2}^{+}\right)\right]-\alpha v_{h}\left(x_{j+1 / 2}^{-}\right) \\
& +\alpha v_{h}\left(x_{j-1 / 2}^{+}\right)=0,
\end{aligned}
$$

where $\alpha$ is arbitrary constant. Hence, the TVD-RK DG with the WAF scheme preserves exactly the stationary solution at steady state. 
TABLE 1: RMS errors when $N=0, N=1$, and $N=2$.

\begin{tabular}{lcccc}
\hline$N$ & Numerical flux & $K=50$ & $K=100$ & $K=200$ \\
\hline \multirow{2}{*}{$N=0$} & HLL & 0.0280 & 0.0215 & 0.0165 \\
& WAF & 0.0182 & 0.0127 & 0.0094 \\
\hline \multirow{2}{*}{$N=1$} & HLL & 0.0130 & 0.0102 & 0.0072 \\
& WAF & 0.0107 & 0.0093 & 0.0067 \\
\hline \multirow{2}{*}{$N=2$} & HLL & 0.0103 & 0.0079 & 0.0053 \\
& WAF & 0.0100 & 0.0076 & 0.0052 \\
\hline
\end{tabular}

Remark 3. One can show that the developed well-balanced TVD-RK DG with WAF scheme in the case of existing source term is consistent, by showing that the approximation of the source term is also consistent.

\section{Numerical Results}

In this section, various test cases have been investigated to demonstrate the accuracy of the present scheme, not only steady, but also unsteady flows.

4.1. Dam Break Flow. It has been shown in the previous section that the weighted average flux is consistent with the TVD-RK DG method. We apply this modified scheme to solve the shallow water equations without source terms in this subsection. The accuracy of numerical solutions is shown and compared with the standard TVD-RK DG when using the HLL method.

The computational domain is that $-5 \leq x \leq 5$. The initial water depth is given by

$$
h(x, t)= \begin{cases}1, & \text { if } x \leq 0 \\ 0.6, & \text { if } x>0\end{cases}
$$

The initial velocity is assumed to be zero. The boundary conditions are transmissive boundaries. We perform 50, 100, and 200 cells in the numerical experiments. Polynomial degree zero, one, and two are applied as a local basis in the TVD-RK DG method. Simulation time is $t=2$, with $\Delta t=$ 0.005. The root mean squared errors (RMS) are shown in Table 1.

When $N$ and $K$ are fixed, the accuracy of numerical solution obtained from the WAF is higher than those obtained from the HLL method. The RMS errors decrease as $K$ increases.

If we fix $K$ and vary $N$, the RMS error decreases as the polynomial degree increases.

The water depth profiles using the HLL and the WAF methods at $t=2$ when $N=1$ and $K=100$ are shown in Figures 1 and 2, respectively. The front of moving shock can be captured correctly by the HLL and the WAF methods. But the scheme using the WAF can capture shock and rarefaction wave more precisely than those using the HLL method. This investigation shows the accuracy of the TVD-RK DG with the WAF method. It has been generalized from the finite volume method.

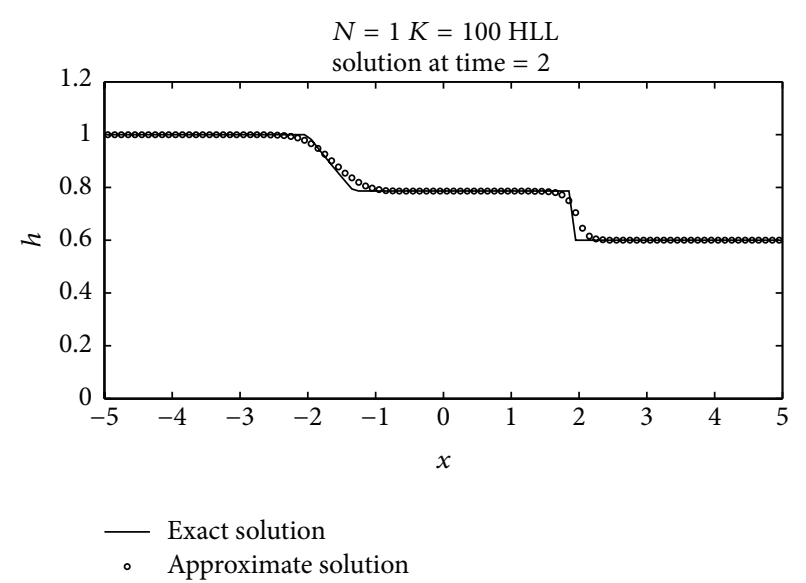

FIGURE 1: Exact solution and water depth profile obtained by the TVD-RK DG HLL method.

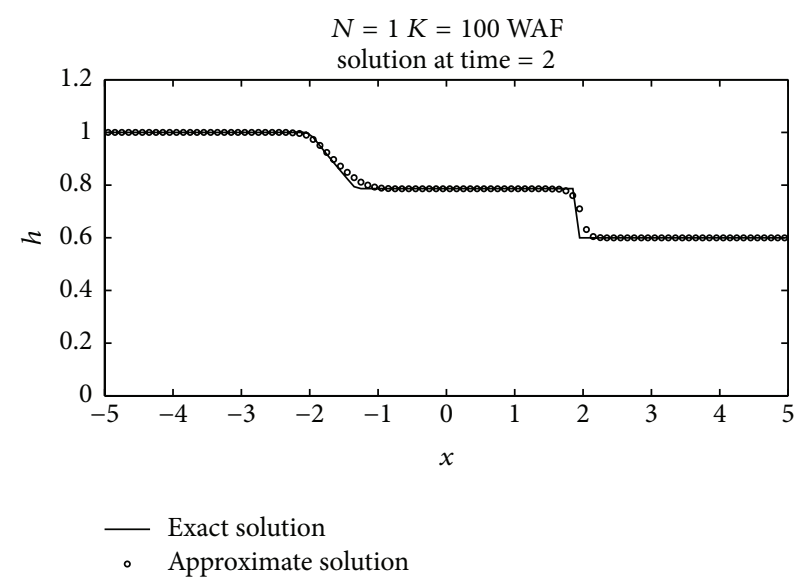

Figure 2: Exact solution and water depth profile obtained by the TVD-RK DG WAF method.

4.2. Flow over Irregular Bed. The uniform channel is length of $1500 \mathrm{~m}$. The bottom elevation is irregular that is shown in Figure 3. This problem is proposed by [5] for testing the accuracy of numerical scheme at stationary state. The boundary conditions are transmissive. The initial water depth is that $h+b=16$, with zero initial velocity. We set $\Delta t=0.01$ and run simulation until $t=100$.

It can be seen from Figure 3 that the well-balanced scheme (dot) gives exactly the stationary solution while the non-well-balanced scheme (dash line) gives solution error especially in the high gradient area of bottom elevation.

4.3. Steady Flow over a Bump. We consider the shallow water flows over a bump in a rectangular channel with length of $25 \mathrm{~m}$. The bump elevation is given by

$$
b(x)= \begin{cases}0.2-0.05(x-10)^{2}, & \text { if } 8<x<12 \\ 0, & \text { otherwise }\end{cases}
$$




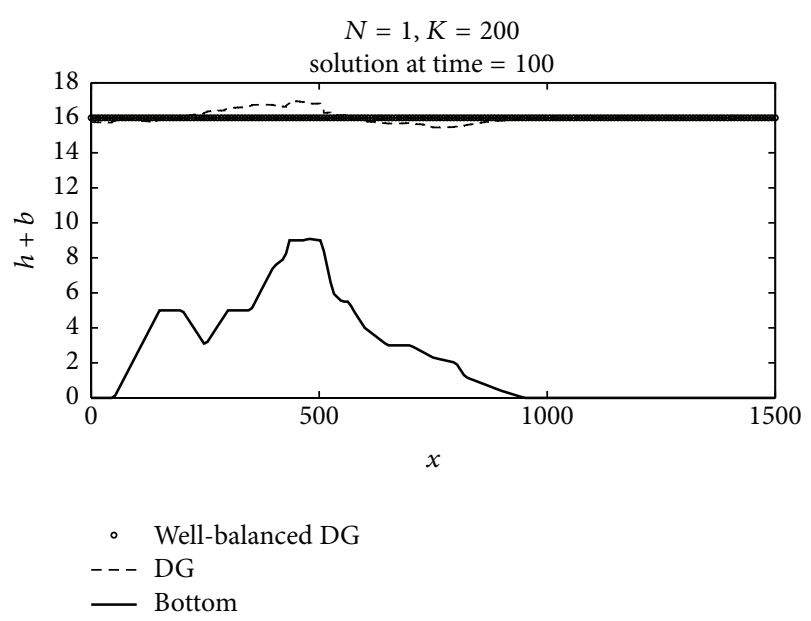

FIGURE 3: Stationary flow over irregular bed by well-balanced and non well-balanced TVD-RK DG with WAF method.

TABLE 2: RMS errors when $N=1$ for subcritical flow over a bump.

\begin{tabular}{lcccc}
\hline & $K=50$ & $K=100$ & $K=200$ & $K=400$ \\
\hline HLL & $1.6954 e-03$ & $4.5563 e-04$ & $1.2546 e-04$ & $3.5900 e-05$ \\
WAF & $1.5816 e-03$ & $4.1648 e-04$ & $1.1474 e-04$ & $3.3019 e-05$ \\
\hline
\end{tabular}

At steady state, classical flows can be characterised by subcritical flow, transcritical flow with shock, and transcritical flow without shock. The TVD-RK DG with the WAF method is performed to solve these problems. The accuracy of numerical solutions can be checked by comparing with the existing analytical solutions; see [20].

Subcritical Flow over a Bump. The upstream boundary is imposed by $q=4.42 \mathrm{~m}^{2} / \mathrm{s}$, while the downstream boundary is set by $h=2 \mathrm{~m}$. The initial water depth is $h+b=2$ with zero initial velocity. Time step is that $\Delta t=0.01$. The RMS errors obtained from the HLL and the WAF methods are shown in Table 2. This shows that the RMS errors obtained by the TVDRK DG WAF are less than those obtained by the HLL for all K.

The water depth and the bump profiles are shown in Figure 4 . The numerical solution is very close to the analytical solution. We also found in this test case that the well-balanced scheme converges to the steady solution faster than the scheme that is not well-balanced.

Transcritical Flow with Shock over a Bump. The upstream boundary is given by $q=0.18 \mathrm{~m}^{2} / \mathrm{s}$, while the downstream boundary is set by $h=0.33 \mathrm{~m}$. The initial condition is that $h+b=0.33 \mathrm{~m}$. The comparison of water surfaces is shown in Figure 5. The numerical result is in good agreement with the analytical result. This shows the accuracy of the wellbalanced scheme that can capture the shock front without any oscillations. It is also found that the well-balanced scheme converges to the steady solution faster than the non-wellbalanced scheme.

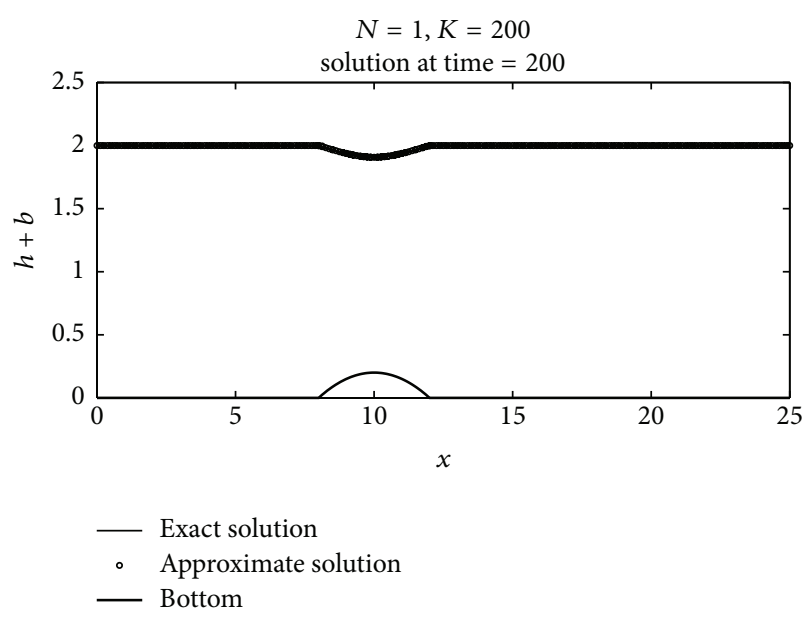

FIGURE 4: Water depth and bump profiles for subcritical flow.

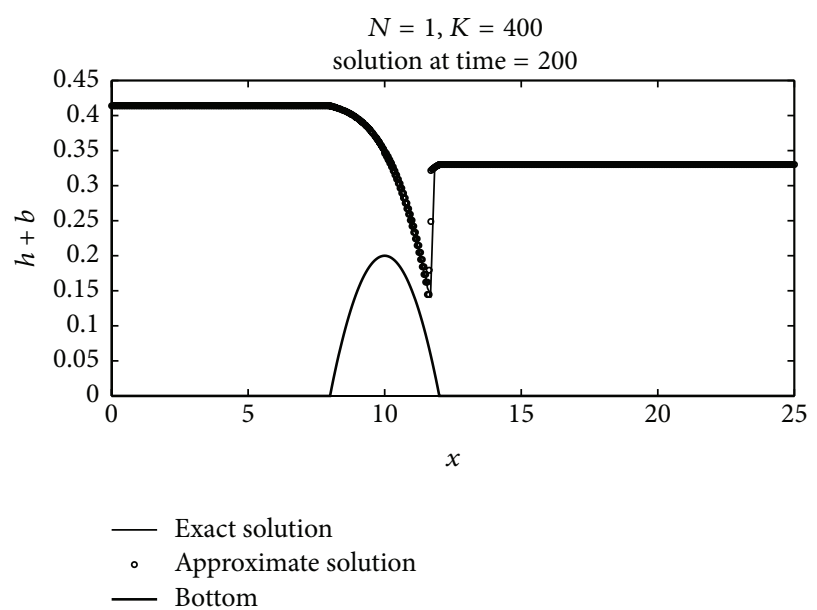

Figure 5: Transcritical flow with shock over a bump.

Transcritical Flow without Shock over a Bump. The upstream boundary is prescribed by $q=1.53 \mathrm{~m}^{2} / \mathrm{s}$, while the downstream boundary is not specified. The initial conditions is that $h+b=0.4 \mathrm{~m}$ with zero initial velocity.

The water depth profiles are shown in Figure 6. The numerical result agrees well with the analytical solution. These results show the accuracy of the well-balanced scheme for solving transcritical flow problem.

4.4. Small Perturbation of Steady State Water. This problem is first proposed by $[18,21,22]$. It can be used to study the capability of numerical scheme for solving small perturbation in shallow water flow. The bottom topography is defined by

$$
\begin{aligned}
& b(x) \\
& = \begin{cases}0.25(\cos (10 \pi(x-1.5))+1), & \text { if } 1.4<x<1.6, \\
0 . & \text { otherwise. }\end{cases}
\end{aligned}
$$




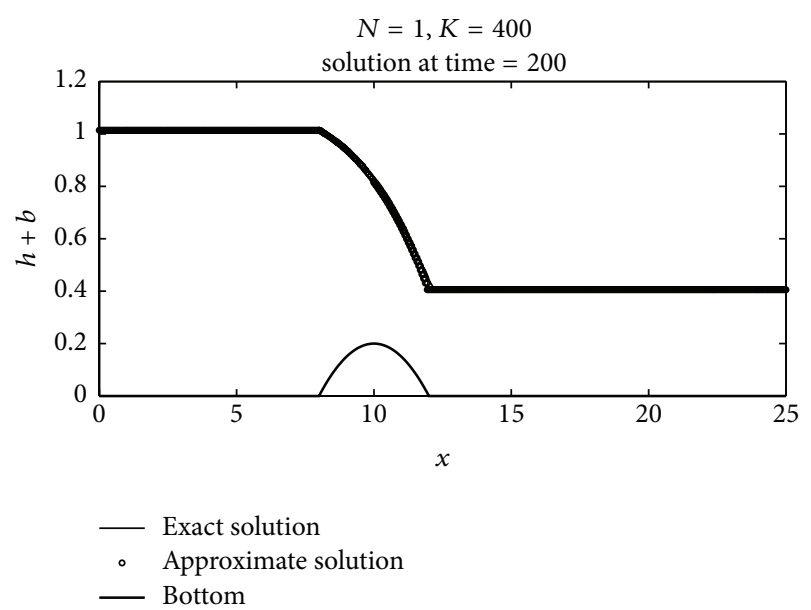

FIGURE 6: Transcritical flow without shock over a bump.

The initial conditions are specified by

$$
\begin{aligned}
& q(x, 0)=0, \\
& h(x, 0)= \begin{cases}1-b(x)+\epsilon, & \text { if } 1.1<x<1.2, \\
1-b(x), & \text { otherwise, }\end{cases}
\end{aligned}
$$

where $\epsilon$ is a nonzero perturbation constant. The boundary conditions are transmissive boundaries. In this work, we consider the cases of $\epsilon=0.2$ and 0.01 . The disturbance of initial water depth from small $\epsilon$ should split the initial wave into two waves. They propagate to the left and the right with characteristic speed $\pm \sqrt{g h}$ at the early stage. A standard scheme which is not well-balanced usually faces with some difficulties to capture correctly the wave speed.

In our simulation, we use 400 uniform grid cells and polynomial degree one in the TVD-RK DG with the WAF method. The simulation time is that $t=0.7$.

The comparison of water depths between our results and the Leveque's solutions is shown in Figures 7 and 8 for $\epsilon=0.01$ and 0.2 , respectively. They are in good agreement for both amplitude and wave speed. These test cases show the ability of our numerical scheme for solving the quasistationary flow with initial disturbance.

4.5. Flow over Nonhorizontal Bed. This test case is presented by [8]. The main propose is to study the ability of numerical scheme for solving unsteady flow over topography. The uniform channel is length of $30 \mathrm{~m}$. The bed elevation is defined by

$$
b(x)= \begin{cases}0, & \text { if } 0 \leq x \leq 10 \\ 0.1(x-10), & \text { if } 10 \leq x \leq 20 \\ 1, & \text { if } 20 \leq x \leq 30\end{cases}
$$

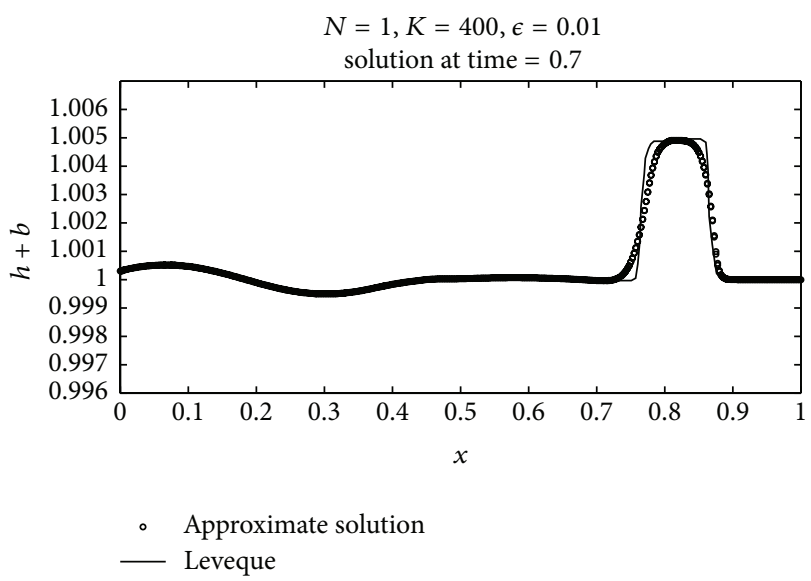

Figure 7: Quasi-stationary flow when $\epsilon=0.01$.

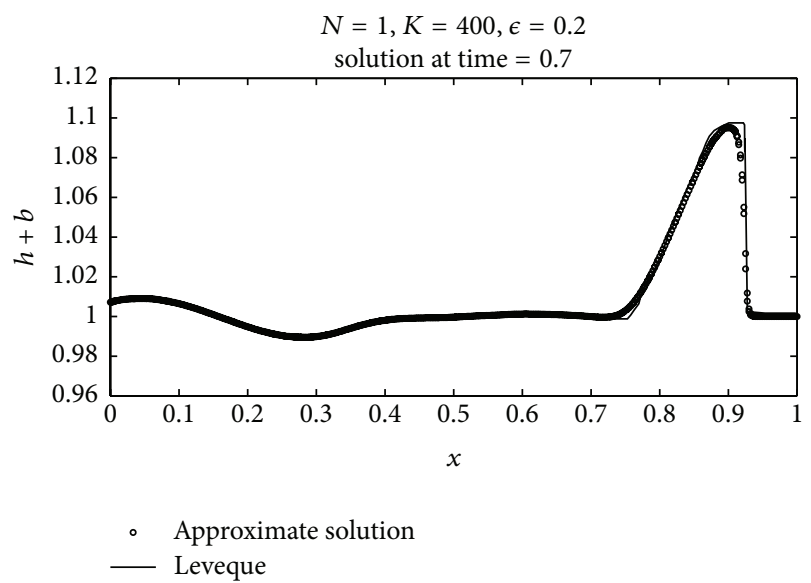

FIgURE 8: Quasi-stationary flow when $\epsilon=0.2$.

The initial conditions are given by

$$
h(x)+b(x)= \begin{cases}4, & \text { if } 0 \leq x<5 \\ 2, & \text { if } 5 \leq x \leq 30\end{cases}
$$

Here, we set $\Delta t=0.01$ and use 200 uniform grid cells with polynomial degree one to simulate the unsteady flow. The boundary conditions are transmissive boundaries.

Our numerical results when comparing with Toro's solutions [8] at $t=1 \mathrm{~s}$ and $4 \mathrm{~s}$ are shown in Figure 9. Wave speed and shock profiles are very close. These results show the accuracy of the present scheme for solving unsteady flow with source term.

\section{Conclusions}

In this work, we present the TVD-RK discontinuous Galerkin method (TVD-RK DG) for solving nonlinear shallow water equations. Most of the TVD-RK DG methods in the literatures usually approximate intercell flux by applying the 


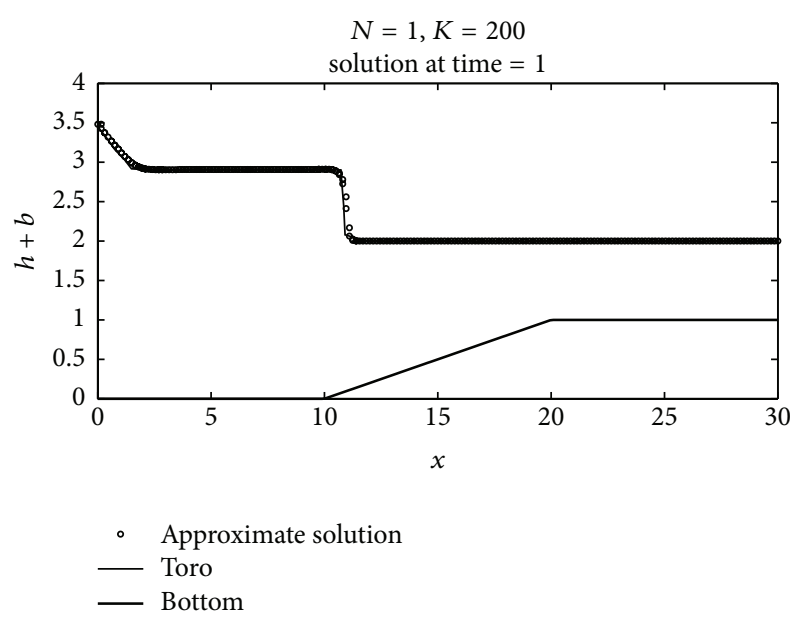

(a)

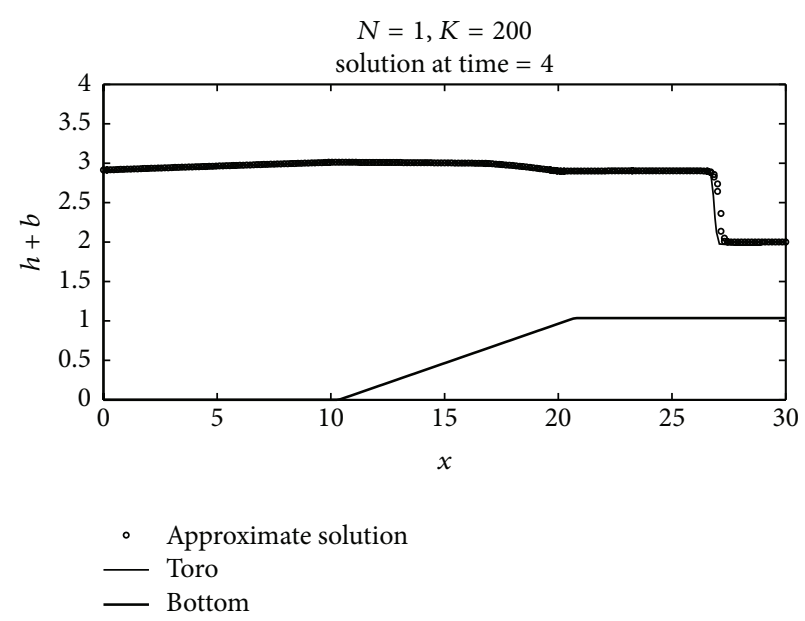

(b)

Figure 9: Flow over non-horizontal bed at time $1 \mathrm{~s}$ (a) and $4 \mathrm{~s}$ (b).

HLL method. But here we apply another approach called the weighted average flux (WAF) in the TVD-RK DG. We have also shown the consistent property of the TVD-RK DG with the WAF approximation. Then the well-balanced TVDRK DG scheme with the WAF approximation is developed. The present method can be used to simulate not only steady flows, but also unsteady flows. The accuracy of modified numerical scheme is demonstrated by various test cases, flow over irregular bed, steady flow over a bump, quasi-stationary, and flow over nonhorizontal bed. The well-balanced TVDRK DG with the WAF method can be used to solve all the kinds of these problems. Moreover, if we restrict at steady state, the scheme using the WAF method converges to the steady solution faster than the scheme using the HLL method. In addition, the well-balanced scheme converges to the steady solution faster than the scheme that is not well-balanced. Due to its advantages of numerical accuracy, simplicity, and wellbalanced property, the present scheme can be modified and extended to simulate two-dimensional problems. However, depending on the types of elements, for example, triangles or rectangles, it is not trivial to extend for two-dimensional problems due to the polynomial basis functions and the WAF fluxes at element interfaces and is not considered in this paper.

\section{Conflict of Interests}

The authors declare that there is no conflict of interests regarding the publication of this paper.

\section{Acknowledgments}

This research is partially supported by the Science Achivement Scholarship Thailand (SAST) to the first author and financially supported by the Thailand Research Fund (TRF) under the Grant no. RSA5680038.

\section{References}

[1] B. Cockburn, C. W. Shu, C. Johnson, and E. Tadmor, Advanced Numerical Approximation of Nonlinear Hyperbolic Equations: Lectures Given at the 2nd Session of the Centro Internazionale Matematico Estivo (C.I.M.E.) held in Cetraro, Italy, June 23-28, 1997, Springer, 1998.

[2] D. Schwanenberg, R. Kiem, and J. Kongeter, "A discontinuous Galerkin method for the shallow-water equations with source terms," in Discontinuous Galerkin Methods: Theory, Computations and Applications, B. Cockburn, G. E. Karniadaki, and C.W. Chu, Eds., Lecture Notes in Computational Science and Engineering, pp. 1419-1424, Springer, 2000.

[3] J. S. Hesthaven and T. Warburton, Nodal Discontinuous Galerkin Methods: Algorithms, Analysis, and Applications, Springer, 2008.

[4] P. A. Tassi and C.-A. Vionnet, "Discontinuous Galerkin method for the one dimensional simulation of shallow water flows," Mecànica Computational, vol. 22, pp. 2403-2428, 2003.

[5] W. Lai and A. A. Bouchut, "Discontinuous Galerkin method for 1D shallow water flow with water surface slope limiter," International Journal on Civil and Environmental Engineering, vol. 3, pp. 167-176, 2011.

[6] E. F. Toro, Riemann Solvers and Numerical Methods for Fluid Dynamics: A Practical Introduction, Springer, Berlin, Germany, 1999.

[7] E. F. Toro, Shock-Capturing Methods for Free-Surface Shallow Flows, John Wiley \& Sons, New York, NY, USA, 2001.

[8] E. F. Toro, "Riemann problems and the WAF method for solving the two-dimensional shallow water equations," Philosophical Transactions of the Royal Society of London A: Mathematical, Physical and Engineering, vol. 338, no. 1649, pp. 43-68, 1992.

[9] M. Maleewong, "Modified predictor-corrector WAF method for the shallow water equations with source terms," Mathematical Problems in Engineering, vol. 2011, Article ID 178491, 17 pages, 2011.

[10] E. F. Toro and P. L. Roe, "A hybridised high-order random choice method for quasi-linear hyperbolic systems," in Proceedings of the 16th International Symposium on Shock Tubes and Waves, p. 701, Gronig, Aachen, Germany, July 1987. 
[11] S. J. Billett and E. F. Toro, "On WAF-type schemes for multidimensional hyperbolic conservation laws," Journal of Computational Physics, vol. 130, no. 1, pp. 1-24, 1997.

[12] A. Bermudez and M. E. Vazquez, "Upwind methods for hyperbolic conservation laws with source terms," Computers \& Fluids, vol. 23, no. 8, pp. 1049-1071, 1994.

[13] G. Kesserwani and Q. Liang, "Well-balanced RKDG2 solutions to the shallow water equations over irregular domains with wetting and drying," Computers \& Fluids, vol. 39, no. 10, pp. 2040-2050, 2010.

[14] Y. Xing and C.-W. Shu, "High order well-balanced finite volume WENO schemes and discontinuous Galerkin methods for a class of hyperbolic systems with source terms," Journal of Computational Physics, vol. 214, no. 2, pp. 567-598, 2006.

[15] E. Audusse, F. Bouchut, M.-O. Bristeau, R. Klein, and B. Perthame, "A fast and stable well-balanced scheme with hydrostatic reconstruction for shallow water flows," SIAM Journal on Scientific Computing, vol. 25, no. 6, pp. 2050-2065, 2004.

[16] S. Noelle, N. Pankratz, G. Puppo, and J. R. Natvig, "Wellbalanced finite volume schemes of arbitrary order of accuracy for shallow water flows," Journal of Computational Physics, vol. 213, no. 2, pp. 474-499, 2006.

[17] C.-W. Shu, "Total-variation-diminishing time discretizations," SIAM Journal on Scientific and Statistical Computing, vol. 9, no. 6, pp. 1073-1084, 1988.

[18] R. J. Leveque, Finite Volume Method for Hyperbolic Problem, Cambridge University Press, 2005.

[19] E. D. Fernández-Nieto and G. Narbona-Reina, "Extension of WAF type methods to non-homogeneous shallow water equations with pollutant," Journal of Scientific Computing, vol. 36, no. 2, pp. 193-217, 2008.

[20] O. Delestre, C. Lucas, P.-A. Ksinant et al., "SWASHES: a compilation of shallow water analytic solutions for hydraulic and environmental studies," International Journal for Numerical Methods in Fluids, pp. 346-365, 2012.

[21] R. J. Leveque, Numerical Methods for Conservation Laws, Birkhäuser Press, 1992.

[22] R. J. LeVeque, "Balancing source terms and flux gradients in high-resolution Godunov methods: the quasi-steady wavepropagation algorithm," Journal of Computational Physics, vol. 146, no. 1, pp. 346-365, 1998. 

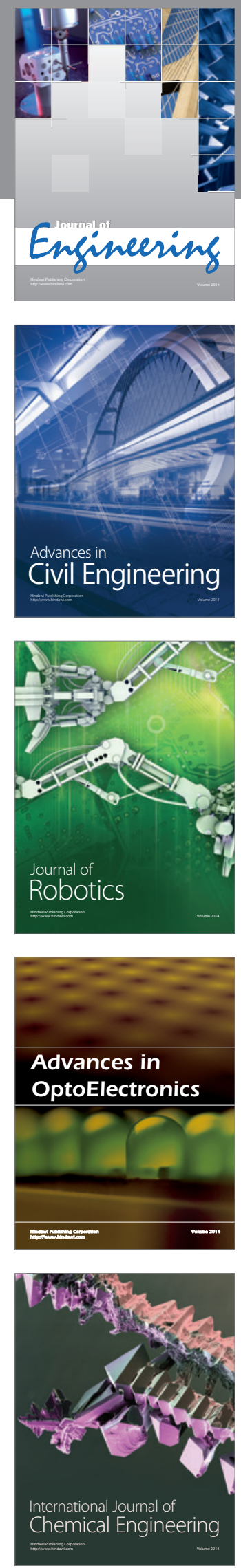

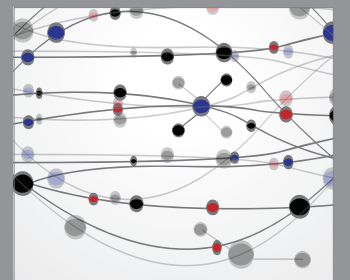

The Scientific World Journal
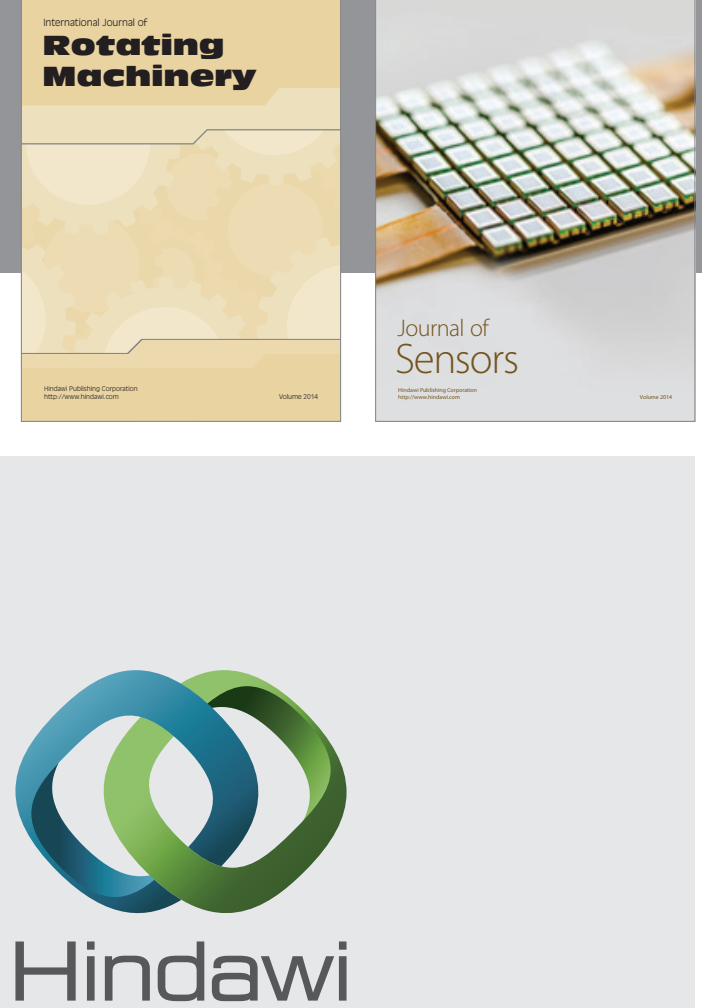

Submit your manuscripts at http://www.hindawi.com
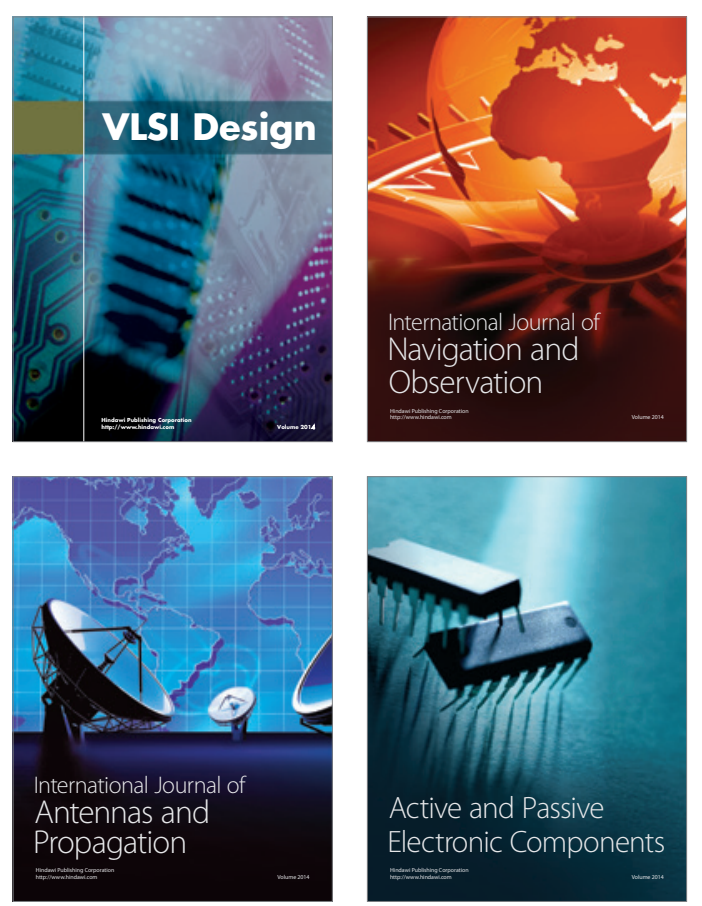
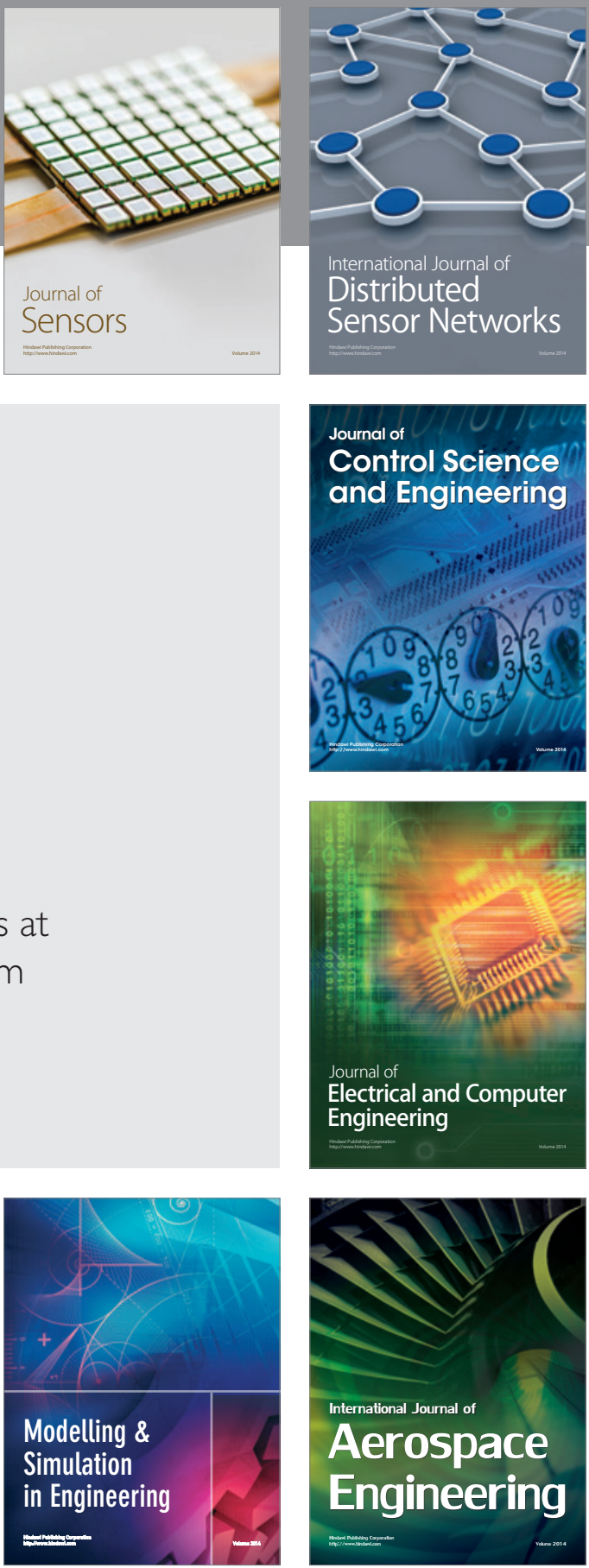

Journal of

Control Science

and Engineering
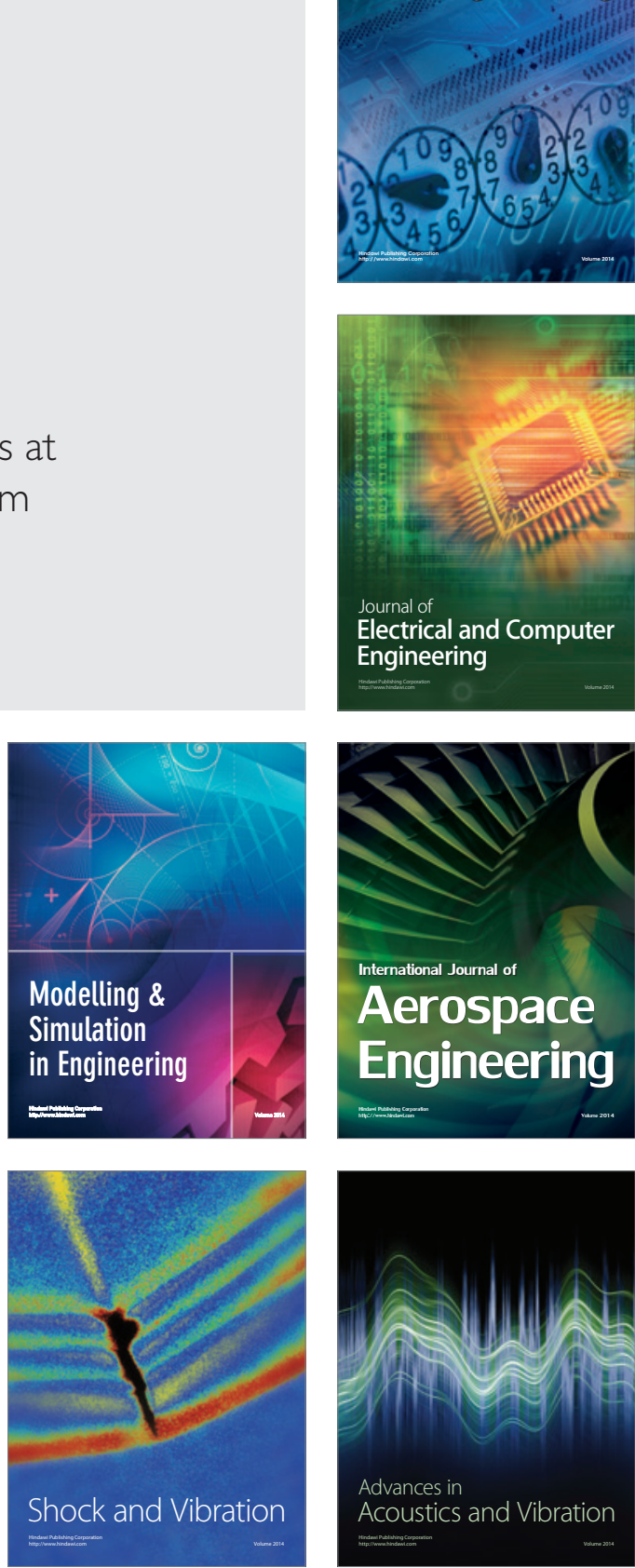